\title{
Perché ci vogliono due lingue per scrivere una poesia?
}

\section{Eva Taylor}

\section{(2) OpenEdition}

1 Journals

\section{Edizione digitale}

URL: https://journals.openedition.org/cher/11206

DOI: 10.4000/cher.11206

ISSN: 2803-5992

\section{Editore}

Presses universitaires de Strasbourg

\section{Edizione cartacea}

Data di pubblicazione: 30 juin 2013

Paginazione: 103-111

ISBN: 978-2-35410-054-4

ISSN: 1968-035X

Notizia bibliografica digitale

Eva Taylor, «Perché ci vogliono due lingue per scrivere una poesia?», reCHERches [Online], 10 | 2013, online dal 08 février 2022, consultato il 10 février 2022. URL: http://journals.openedition.org/cher/ 11206 ; DOI: https://doi.org/10.4000/cher.11206

\section{(c) (i) (8)}

Ce(tte) œuvre est mise à disposition selon les termes de la Licence Creative Commons Attribution Pas d'Utilisation Commerciale - Partage dans les Mêmes Conditions 4.0 International. 


\title{
Perché ci vogliono due lingue per scrivere una poesia?
}

\author{
EVA TAYLOR \\ Scritttice
}
Ho due bocche
da una parlo
dall'altra sanguino.
Stamattina ho scelto il rossetto più rosso
per coprire le tracce di sangue.
Mi hai guardato e hai detto:
stai bene.

Ta domanda del titolo non ha nulla di retorico per chi la pone. Nasce Lda una costellazione linguistica in cui si trova chi non scrive nella sua lingua madre, vivendo con due (o più) lingue. Cercherò di dare una prima risposta con una breve poesia. Si tratta del numero 22 della sezione Prima igiene, che fa parte di una serie di composizioni pubblicate dalle Edizioni l'Obliquo di Brescia nel 2006 sotto il titolo L'igiene della bocca':

Una piccola scena quotidiana: lei si trucca e lui la guarda e approva. Ma lei sa che sotto il trucco nasconde qualcosa, qualcosa che sanguina, forse un piccolo taglio, forse una ferita, il testo non lo dice, parla piuttosto di due bocche: «da una parlo, dall'altra sanguino». C'è l'immagine di una bocca che soffre, che non riesce a dire direttamente in parole quello che vuole dire, c'è come un resto che rimane inespresso. Ecco l'immagine della bocca che sanguina, perché non trova le parole ma provoca tensione. Da questa tensione nasce la breve poesia, dalla tensione di non poter comunicare appieno ciò che si vorrebbe dire. Credo che si tratti di un'esperienza molto

1 E. Taylor, 22 (Sezione Prima igiene), in L'igiene della bocca, con una nota di A. M. Carpi, Brescia, Edizioni L'Obliquo, 2006, p. 30. 
comune, c'è sempre qualcosa che non siamo riusciti a dire. Ha scritto Anna Maria Carpi nella sua nota al libro: «è chiaro che in questione non è la bocca, bensì il sapere, il sentire che, in qualsiasi lingua, il vuoto si occulta sotto vane conversazioni, quelle che facciamo tutti i giorni per campare»².

Quindi una prima risposta alla domanda che mi sono posta è proprio questa: per scrivere una poesia ci vuole una lingua diversa dalla lingua delle «vane conversazioni». In questo senso tutti gli scrittori hanno bisogno di due lingue per scrivere una poesia. Ma che cosa cambia in questa situazione per chi scrive in una lingua che non è la sua prima lingua? La mia lingua materna è il tedesco, una lingua che spesso non riscuote molto entusiasmo. Cercate di immaginarvi solo per un momento di aver il tedesco come madrelingua, di averla in bocca, di parlare il tedesco ad altre persone, forse potete sentire quella tensione tra le due bocche di cui nella poesia citata sopra. Non perché il tedesco sia una lingua particolarmente difficile, ma perché questa lingua porta ancora un peso storico-culturale che non è svanito. Voi mi direte, ma no, esageri, non è così. Lo metto in conto e nonostante ciò sento l'eredità della mia lingua come una contraddizione che si crea tra la bellezza e straordinaria leggerezza delle poesie di Heinrich Heine per esempio e il suono della voce di chi comandava e di chi ha poi costruito un muro per dividere il paese. Non era la lingua, sia chiaro, ma erano le voci degli esseri umani che ab-usavano della lingua. È una contraddizione che mi ha portato a sentire i dolori in bocca, quella bocca che non si vede, ma che sanguina.

Credo che oggi la lingua tedesca sia in una situazione completamente diversa; è una lingua che vive un momento di contatto linguistico intenso, prima di tutto con l'inglese, ma il contatto linguistico va ben oltre, riguarda anche altre lingue di gruppi migranti, i turchi, i russi o anche gli italiani. È quindi possibile che una lingua - come il tedesco - si rinnovi proprio in questo processo di contatto linguistico e che sia possibile seguire una nuova letteratura che nasce da questa costellazione e che ha un'enorme importanza. Ho capito la mia situazione «in bocca» cominciando a scrivere in italiano, che invece di essere mia seconda lingua è diventata la mia prima bocca (per rimanere in metafora), che mi ha permesso di raccontare anche alcuni momenti della mia storia «tedesca». A testimonianza di questo riporto qui una poesia dal titolo La casa del nonno ${ }^{3}$ :

Nei muri le crepe

le finestre spalancate

2 Ibidem, p. 58.

3 Ead., La casa del nonno, in Volti di parole, Brescia, Edizioni L'Obliquo, 2010, p. 14. 
sul prato il bianco delle oche il tiglio piantato quel giorno.

Sento il racconto degli ultimi anni

le notti senza sonno e le lettere

i viaggi, le attese e la rabbia.

Io guardo

ma tu sull'altro lato della strada

in questo luogo dove sei nato

non torni più

per una virgola di legge

messa a Mosca

firmata a Berlino.

L'ombra del tiglio abbraccia noi due

ma tu non ti riposi

ed io ne sfuggo.

Tramite la scrittura in italiano ho affrontato momenti della mia storia, che però non è solo storia personale, ma ovviamente è solo una scheggia della storia generale. E in questo raccontare, seppur in forma lirica, mi sono riavvicinata anche al tedesco come lingua espressiva. Non solo ho (ri)cominciato a scrivere anche in tedesco, ma è come se avessi sentito il bisogno di usare il tedesco anche scrivendo in italiano. Questo ha portato a un contatto linguistico nelle poesie stesse. Per esempio nel breve ciclo Tattoos $^{4}$ ricorrono titoli in tedesco e sfrutto il principio della composizione nella formazione delle parole, che come è noto in tedesco è particolarmente fertile. Vengono così evocate immagini che riguardano la pelle e giocano con la possibilità di creare nuove parole e quindi nuove immagini come il titolo di un componimento, Kleidleid', che significa letteralmente 'vestitosofferenza', dove l'immagine del vestito viene associata al fastidio quotidiano del corpo quando si trova in un involucro troppo stretto:

la stoffa perforata

vuota di carne

coperta di squame

respiro di pelle

non di bocca

ho voluto espirare

un'altra me

4 Ibidem, p. 35-40.

5 Ibidem, p. 38. 
fare una A da una $\mathrm{E}$

senza costola

solo coll'ago

delle lettere

sfila questo filo di smarrimento

la linea senza tracce

sciogli le carte antiche

gli spazi bianchi

infilati

ricucimi

sulla prossima pagina.

È stato quindi l'italiano a spingere la mia scrittura anche in tedesco e alla fine si è messo in moto un reciproco processo di auto-traduzione, che mi permette di rivedere alcune poesie sia in italiano sia in tedesco e di sviluppare quindi una doppia prospettiva. L'autotraduzione è stata nella maggior parte dei casi una riscrittura, che mi ha dato vere lezioni di lingua (si potrebbe anche dire forse di poetica), in cui lo spostamento delle parole e la loro ritessitura hanno portato alla luce spazi espressivi prima non definiti. $\grave{E}$ un processo che va in tutte e due le direzioni, sia dall'italiano in tedesco che viceversa.

Scrivere in una lingua diversa dalla madrelingua non significa automaticamente anche autotradursi. Nell'autotraduzione l'autrice dell'originale coincide con la traduttrice e quindi potrebbe essere più legata alla prima lingua e ad aspetti formali e stilistici che sono tipici di questa. Quando si scrive nella lingua due, si cambia binario linguistico e di conseguenza c'è uno scarto anche nell'immedesimazione nella lingua nella quale si accinge a scrivere, cercando quasi di lasciarsi dietro la lingua materna. Che questa possa riaffiorare in maniera piuttosto inconsapevole nelle strutture e figure linguistiche e che si verifichino delle interferenze continue è un fenomeno dovuto a questo intimo collegamento. Nell'autotraduzione si crea un dialogo interlinguistico. Chi scrive entra in un interspazio che porta a un costante cambiamento di prospettiva. Si tratta di una mediazione interna e porta a due testi: l'originale e la sua traduzione sono in un certo senso testi gemelli, ma non sono gemelli monozigoti. Si sposta quindi il registro e si fanno scoperte interessanti. Vorrei portare due esempi iniziando con le due versioni, in tedesco e in italiano, della prima poesia del ciclo 'Gartenarbeit', ovvero 'Giardinaggio': 
1

Mich fing ein Herz

mich griff ein Grün

drang warm in meine Spitzen.

Und seine Art war

tief zu schlafen

im Leben und in mir.

Gedieh ich eng,

gedieh ich weit

trieb's Blätter aus Papier.

Mich schnitt ein Herz

mich brach ein Wort

und auf dem Beet hier:

ausgelaufnes Blau.

E. Taylor, 1 - Sezione Gartenarbeit, in Gartenarbeit, Bordenau-Venezia, San Marco Handpresse, 2010.
1

Un cuore m'ha presa un verde $m$ 'ha afferrata fino alle mie punte il suo calore.

Un sonno profondo

a modo suo

dormiva

nella vita e in me.

Venivo su poco

venivo su molto

sempre crescevano

foglie di carta.

Un cuore mi ha tagliata

una parola mi ha spezzata

e qui sull'aiola:

l'inchiostro si rovescia.

Ead., 1 - Sezione Giardinaggio, in Volti di parole, cit., p. 55.

Nel testo tedesco ci sono delle ripetizioni ritmiche (mich fing ein Herz/ mich griff ein Grün// gedieh ich eng/gedieh ich weit// mich schnitt ein Herz/ mich brach ein Wort) che difficilmente si possono trasportare in italiano. Ma come altro elemento di costruzione del testo ci sono le allitterazioni Blau ('azzurro/blu') e Blatt ('foglio/foglia'). Quest'ultima parola necessita inoltre di essere disambiguata in italiano e nell'autotradurmi, dovendo scegliere tra 'foglio' e 'foglia', ho proseguito nella dimensione del giardino, l'immagine che tiene insieme tutta la sezione. Ho scelto quindi la versione «foglie di carta», che mi sembrava più incisiva rispetto al semplice "fogli di carta». Per quanto riguarda invece la parola blau, c'è da dire che in tedesco è colore che contiene delle sfumature e per le quali in italiano esistono delle parole distinte, ovvero blu, azzurro, ecc. Anche qui si pone quindi la domanda di quale termine scegliere per l'italiano. Inoltre bisogna considerare che blau in tedesco è un colore mentale, legato al Romanticismo, alla simbologia della «Sehnsucht», del desiderio, una dimensione astratta che in italiano è difficile da rendere nella scelta tra «azzurro» e «blu». Nella traduzione in italiano mi sono attenuta quindi di nuovo alla cornice semantica del giardinaggio, concretizzando la parola blau coll'immagine dell'inchiostro, una metonimia della scrittura che sposta il realismo della descrizione in un altro campo semantico. 
Ma la mediazione per il processo di autotraduzione che diventa riscrittura può attraversare tappe più complesse, che vorrei illustrare con questo secondo esempio. Si tratta della poesia Schneebuch ${ }^{6}$ in tedesco e il suo «doppio» in

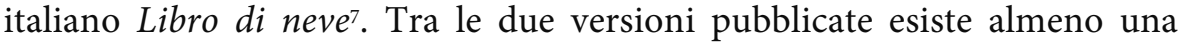
versione di lavoro, necessaria, ma rimasta nel cassetto, che qui riporto come forma intermedia e che per chiarezza ho chiamato Libro di neve (1).

Schneebuch

In absentia

taste ich Wortlinien nach, zeichne sie ab, wo Sand sich ausbreitet.

(Atlanten aus früh'rem Leben haben mich vom Weg abgebracht.)

Schnee liegt nun auf den Dächern

- Stille und Licht -

in deiner Abwesenheit

finde ich ein Schneebuch.

Dünne Seiten

wie Wellen aus der Tiefe der Haut sprechen von dir

in absentia.
Libro di neve (1)

In assenza

seguo col tatto le linee di parole le copio, dove sabbia si estende. (atlanti di vita passata mi hanno distolta dal cammino).

Neve è sui tetti ora

- silenzio e luce -

nella tua assenza

trovo un libro di neve.

Pagine sottili

come onde dal profondo della pelle parlano di te in assenza.

\section{Libro di neve}

Hai lasciato un libro di neve sul tavolo si sta sciogliendo in piccole gocce le tue parole ad una ad una sul marmo le sento e leggo per tutto l'inverno dei tuoi passi sulla terra di ghiaccio delle storie che racconti con poche parole.

E vedi qui ora la mia bocca solitaria si apre si chiude per dire qualcosa la lingua nel suo silenzio diligente fa cadere lettere sparse e quello che esce è un brontolio.

Di nuovo prendo il libro di neve gli occhi si perdono su pagine-cristalli si vedono gli archi di Strada Maggiore l'immagine sfocata della neve grigia. E l'inverno è breve e la luce è spenta e di poco si allungano le giornate la sera.

6 Ead., Schneebuch, in Aus dem Schneebuch, Bergen / NL, Eric van der Wal, 2008.

7 Ead., Libro di neve, in Volti di parole, cit., p. 9. 
L'originale in tedesco (rispetto alla versione finale in italiano più sintetica) è una specie di meditazione, sviluppata intorno a delle immagini che non pretendono di essere una descrizione, ma rimangono in qualche maniera luoghi non concreti, rappresentazioni, quello che in tedesco si intende per «Seelenlandschaft», che significa "paesaggio dell'anima». C'è per esempio la metafora della sabbia, legata alla collocazione dei sentieri che si perdono nella sabbia. In tedesco la collocazione «im Sande verlaufen» significa letteralmente "perdersi, smarrirsi nella sabbia», ma anche qualcosa che si perde nel nulla, che si insabbia, e ricorda l'espressione italiana «sabbie mobili». C'è quindi l'espressione di un senso di smarrimento, un non saper dove andare, perché le linee di un vecchio atlante non corrispondevano più a un percorso giusto, portavano su un sentiero sbagliato. Quest'immagine viene resa nella versione di lavoro Libro di neve (1).

La prima versione tradotta in qualche maniera però non rendeva la dimensione sospesa di ciò che il tedesco conteneva. Mi sembrava come troppo scarna, come se mancasse qualcosa. Ho cominciato a riscriverla a partire dal titolo un giorno a Bologna, quando nevicava e ho provato la sensazione di fiocchi di neve sulla mia pelle - una sensazione che mi ha rimesso in contatto con la poesia in una maniera molto vitale.

L'ultima versione - quella in italiano dal titolo Libro di neve - è più narrativa, con precise indicazioni anche del luogo, non solo di un luogo esterno come Strada Maggiore a Bologna, ma anche di un luogo interno, una stanza con un tavolo di marmo (forse una cucina), come se la poesia dovesse essere radicata in un luogo. In tutte e tre le poesie c'è la metafora del libro di neve, rimasta anche nei titoli, nella prima versione in tedesco l'io lirico trova un libro di neve che parla dell'altro in assenza (è come se parlassi del ritrovamento di una lingua), nell'ultima poesia il libro di neve è legato a un 'tu' che ha lasciato il libro, quindi il libro è un dono preciso, anche se le sue parole svaniscono come nella prima poesia, perché sono fatte di neve; in tutte le versioni il libro di neve è un libro dei ricordi.

Se dovessi sintetizzare la differenza tra le versioni 'finali', quella tedesca e quella italiana, direi che tutte e due girano intorno all'immagine del sentiero, una strada da fare, trovare, proseguire: mentre nella versione in tedesco l'enfasi è posta più sullo smarrimento del sentiero, nella versione italiana l'accento si sposta sul ritrovamento del sentiero. Sono quindi due movimenti opposti, ma complementari.

Scrivere in due lingue per me apre questa possibilità: rivedere i testi nel tempo; la riscrittura assume più volte la forma di un ridisegno, con 
cambiamento di prospettiva, di toni e colori, quindi spostamenti anche minimi, senza arrivare mai a una fine, perché ogni nuova versione ne richiama una nuova nella rilettura nell'altra lingua.

La traduzione diventa allora una specie di cartina di tornasole per rilevare non solo le intenzioni che stanno dietro alla parola, ma anche le potenzialità che si possono sviluppare in un'altra lingua. Una specie di liquido rivelatore, o di sviluppatore (come le bacinelle con i liquidi nelle vecchie camere oscure, con l'immagine che affiora lentamente, dal negativo al positivo).

In questo percepire i processi di tessitura, nel fare e disfare, c'è una continuità che ha qualcosa dei lavori in casa. Forse non a caso così facendo sono arrivata a scrivere un Ricettario minuto ${ }^{8}$. Con questo "ricettario» ho seguito in un certo senso un principio simile a quello di Igiene della bocca. Allora era il fascino delle parole tecniche, del lessico odontoiatrico, parole che anche per chi conosce bene una lingua rimangono sempre un piccolo mistero. Nella lingua non materna c'è un maggiore effetto di straniamento e anche una minore consuetudine con certe parole. Me ne sono servita per esprimere la tensione tra le due bocche e di cui ho parlato all'inizio. Esempi dai due cicli, in cui è evidente l'impiego la rielaborazione di strutture e terminologia caratteristiche dei linguaggi specialistici, sono le due poesie che seguono, Bruxismo e Ricetta per un pesce fuor d'acqua ${ }^{10}$ :

La mattina mi dici:

di notte c'era un rumore stridente in tutta la casa.

E subito sono colpita da un senso di vergogna appiccicoso.

Nello specchio si vedono chiari segni d'abrasione.

I muscoli dei miei mascellari sono affaticati e dolenti.

Soffro di mal di testa: davanti all'orecchio, sulla guancia e sulla tempia.

Digrignamento e chiusura contratta dei denti:

forme comuni di parafunzioni

di cui la paziente non è consapevole.

Sono virgole, punti, trattini e lettere da stritolare

il contingente di tre lingue in ogni parola

(e anche se non si vede, lo sento in bocca).

E il dolore è sordo, pulsante e continuo.

8 La Sezione Ricettario minuto è contenuta nella raccolta Volti di parole, cit., p. 23-31.

9 E. Taylor, Bruxismo, in L'igiene della bocca, cit., p. 40 (contenuta nella Sezione Igiene alfabetica).

10 Ead., Ricetta per un pesce fuor d'acqua, in Volti di parole, cit., p. 28 (contenuta nella Sezione Ricettario minuto). 
La maggior parte dei pesci è muta

Lasciatelo bollire qualche istante

fatelo raffreddare nell'acqua di cottura

infine versatelo nell'alfabeto.

Imparerà a nuotare, a respirare e a parlare

ma avrà sempre l'impressione

di essere qualcos'altro.

Quel suo lieve sapore amarognolo

che alcuni apprezzano

e altri trovano nauseante

vi suonerà come accento.

Si può togliere con un filo d'olio d'oliva.

Extravergine, macinato a freddo.

Meglio freddissimo. Quasi come d'acqua.

L'intertestualità, in particolare nel secondo testo, si articola non solo a livello lessicale, bensì sul genere testuale, e cioè sulle formule tipiche del ricettario con le sue frasi brevi, descrittive e imperative. La scrittrice Emine Sevgi Özdamar, di madrelingua turca, ma una delle voci più significative della letteratura tedesca, in un romanzo dichiara: «le mie parole in tedesco non hanno un'infanzia» ${ }^{11}$. Con questa affermazione la scrittrice probabilmente vuole indicare una forma di mancanza di pienezza di senso e, forse, di ricordi, di sensazioni. Sono convinta che nel processo di autotraduzione ci sia un avvicinamento intenso tra le lingue, che fa recuperare una dimensione simile a quella del piacere infantile nell'apprendimento della madre lingua e del radicamento che è connesso con questo processo. Le mie parole in italiano nascono da un piacere delle parole, quasi come nell'infanzia.

11 E. Sevgi Özdamar, Die Brücke vom Goldenen Horn, Düsseldorf, Kiepenheuer \& Witsch, 1998 , p. 35. 